from Michael Michailov (ed.), Nanophenomena at Surfaces: Fundamentals of Exotic Condensed Matter Properties (StranskiKaischew memorial) (Springer, Berlin, 2010(??))

\section{Multisite Interactions in Lattice-Gas Models}

\section{T.L. Einstein and Rajesh Sathiyanarayanan}

Department of Physics, University of Maryland, College Park, Maryland 20742-4111 USA einstein@umd.edu

Abstract. For detailed applications of lattice gas models to surface systems, multisite interactions often play at least as significant a role as interactions between pairs of adatoms that are separated by a few lattice spacings. We recall that trio (3adatom, non-pairwise) interactions do not inevitably create phase-boundary asymmetries about half coverage. We discuss a sophisticated application to an experimental system and describe refinements in extracting lattice-gas energies from calculations of total energies of several different ordered overlayers. We describe how lateral relaxations complicate matters when there is direct interaction between the adatoms, an issue that is important when examining the angular dependence of step line tensions. We discuss the connector model as an alternative viewpoint and close with a brief account of recent work on organic-molecule overlayers.

\subsection{Introduction}

A thorough understanding and characterization of surface energetics is important for fabricating nanostructures with desired morphological features. To this end, lattice-gas models have been very successful in categorizing structural properties, energetics and evolution of adatoms and steps on surfaces, as discussed in a variety of reviews [1-4]. They have also been applied to analogous but more complicated systems [5], such as electrochemistry [6]. The underlying viewpoint is that a set of interactions is sufficient to understand both equilibrium and dynamic surface processes. Lattice-gas models provide a powerful and convenient route to explore how microscopic energies influence the statistical mechanics of nanoscale to submonolayer structures on crystalline surfaces. Such models underlie most Monte Carlo (and transfer matrix) simulations. They assume that overlayer atoms (or other adsorbed units) sit at particular high-symmetry sites of the substrate. The parameters of the model are then the interaction energies between such atoms and/or the barriers associated with hops between the high-symmetry positions.

The use of lattice-gas models proceeds in two generic ways. In the first, one posits a few energies that are likely to dominate the physics of interest and then computes with Monte Carlo simulations the desired equilibrium or dynamic properties, deriving thereby the values of these energies from fits $[1,7,8]$. The dangers of this approach are: 1) the properties of interest may be relatively insensitive to the specific interactions and 2) there may be other interactions that are non-negligible, so that the deduced energies are effective rather than actual.

The second approach [9-13] begins by actually computing the (many) energies of importance, a task that is now possible with efficient densityfunctional-theory packages such as VASP (the Vienna Ab-initio Simulation Package) $[14,15]$. This process can be used to compute interaction energies between relatively distant neighbors. One should also compute multi-atom interactions, which can also be significant $[3,16]$. As above, these interactions are then used in Monte Carlo simulations to test whether they account adequately for experimentally observed properties such as phase diagrams, equilibrium island shapes, or step fluctuations. This approach is appealing because the calculated interaction energies can be self-consistently checked for completeness, thereby mitigating the second danger mentioned above. Assuming that one has sufficient computational power to compute all the interactions that contribute at the level of the desired precision, there is still the danger that the interactions depend sensitively on the local environment, making a simple lattice gas description inadequate. These caveats notwithstanding, lattice gas models have been extensively used in the realm of surface physics to describe such diverse phenomena as phase transitions, phase diagrams, equilibrium island shapes, concentration-dependent diffusion, step fluctuations, and growth.

The basic assumptions that underlie lattice-gas models are: (i) all atoms sit at high-symmetry positions and local relaxations produce the final structure, (ii) a finite set of effective interactions is sufficient to understand all the surface processes and (iii) interactions are not sensitive to local positions of the adatoms. In the simplest scenario, only pair interactions between nearest neighbors are considered. However, in certain cases, like the orientation dependence of step stiffness and the equilibrium shape of islands, long-range pair interactions and multi-site interactions are required for a complete description $[8,13,17-21]$. The substrates in these studies are typically mid or late transition or noble metals, where the electronic indirect interaction leads to rich behavior [3].

Explicitly, the lattice-gas Hamiltonian of adatoms on a surface is written as:

$\mathcal{H}=\sum_{m=1}^{M} E_{m} \sum_{\langle i j\rangle_{m}} n_{i} n_{j}+\sum_{T} E_{T} \sum_{\langle i j k\rangle_{T}} n_{i} n_{j} n_{k}+\sum_{Q} E_{Q} \sum_{\langle i j k \ell\rangle_{Q}} n_{i} n_{j} n_{k} n_{\ell}+\ldots$

where $n_{i}$ is the occupancy of the high-symmetry lattice site indexed $i ; n_{i}=1$ denotes an occupied site and $n_{i}=0$ denotes an empty site. Interactions between adatom pairs up to the $\mathrm{m}^{\text {th }}$-neighbor pair $E_{m}$ are included in the model; accordingly, interactions between adatom pairs that are separated by distances 


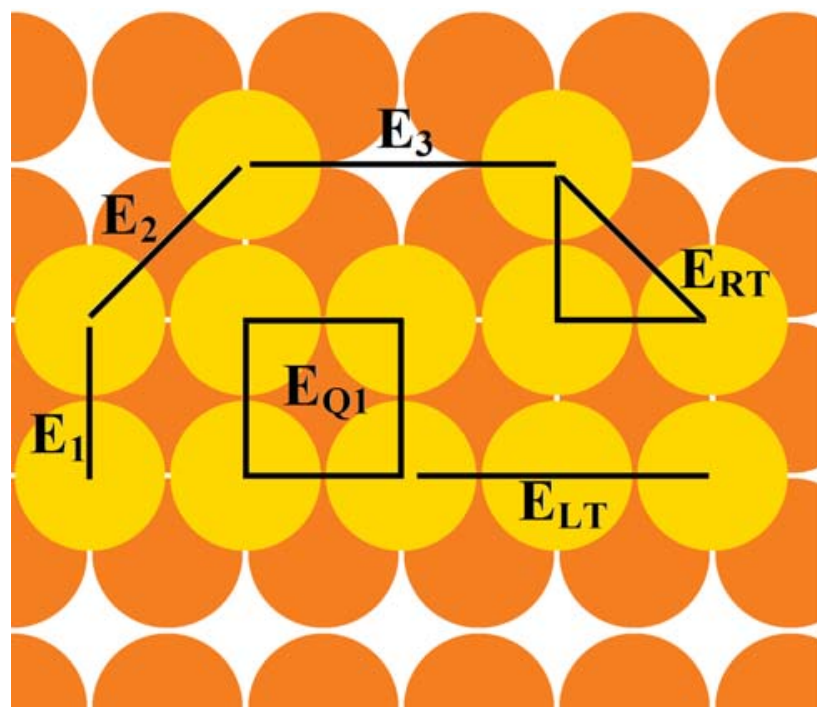

Fig. 1.1. Illustration of $\mathrm{NN}$ (nearest neighbor), NNN (next NN), and $3 \mathrm{NNN}$ (third $\mathrm{NN})$ pairs with interaction energies $E_{1}, E_{2}$, and $E_{3}$, respectively. Also shown are the isosceles right triangle (RT) with energy $E_{R T}$ or, more formally, $E_{112}$ (denoting a pair of NN legs and one NNN leg), and an isosceles linear triangle (LT) with energy $E_{L T}$ or, more formally, $E_{113}$. Lastly, the most compact quarto interaction has interaction energy $E_{Q 1}$ or, more formally, $E_{1111}$. For clarity [and physical relevance], here and in Fig. 1.5, the adatoms are placed in center sites, as for homoepitaxy on the (100) face of an fcc crystal like $\mathrm{Cu}$, rather than atop sites as for homoepitaxy on a simple cubic crystal.

greater than the $\mathrm{m}^{\text {th }}$ neighbor distance are expected to be insignificant. $E_{T}$ stands for three-adatom non-pairwise "trio" interactions, with the index running over all trimer configurations of significant strength. Similarly, $E_{Q}$ stands for four-adatom non-pairwise (and with trios also subtracted) "quarto" interaction. (The possibility of quarto interactions has been known for over three decades $[3,22]$, but, to the best of our knowledge, it has been invoked only once in an actual calculation of adsorbate energetics [23]) until very recently [24,25]. If necessary, pair interactions with a longer range and/or higher-order multisite interactions (possibly, five-adatom quintos) are included in the model till adequate convergence between theoretical predictions and experimental observations is obtained. However, the inclusion of a large number of interaction parameters makes the lattice-gas model intractable, thereby severely undermining the efficacy of lattice-gas models in characterizing overlayer systems.

The nature of the interactions leading to the lattice-gas pair energies have been reviewed extensively $[3,26-28]$ so we will present just a quick summary.

\section{T.L. Einstein and Rajesh Sathiyanarayanan}

If there is charge transfer (workfunction change) during the adsorption process, the adsorbates can interact electrostatically. Other "direct" interactions between the adatoms themselves can occur when the adatoms are at NN (nearest-neighbor) or perhaps NNN (next NN) sites. These are strong interactions, comparable to the binding energy, with the substrate plaving a minor, sometimes negligible role; they are liable to produce relaxations that thwart straightfoward application of lattice-gas models (cf. Section 1.5.2). When adatoms are sufficiently separated that there is insignificant overlap of the electron orbitals, there can still be an "indirect" interaction-weaker but of longer range - mediated by substrate electrons or the elastic field. Elastic interactions tend to be of one sign and decay monotonically with adsorbate separation distance $d$, like $d^{-3}$ asymptotically. They are generally taken to be isotropic, even when unjustified by the elastic tensor, since computing elastic Green's functions in the anisotropic case is notoriously difficult [29]. The electronic indirect interaction has richer behavior, oscillating in sign and reflecting the isotropy or anisotropy of the substrate wavefunctions in the surface plane [30]. Asymptotically it is dominated by the Fermi wavevector and has the Friedel behavior

$$
d^{-n} \sin \left(2 k_{F} d+\Phi\right)
$$

where $d$ is the [lateral] distance between the adsorbates; this result is nonperturbational, the phase factor $\Phi$ distinguishing it from the well-known perturbational RKKY expression [31]. (For a non-isotropic Fermi surface, the appropriate wavevector has velocity parallel to d; see Ref. [3] for details.) In the bulk, $n=3$, but at a metal surface the leading term is cancelled by that from its image charge, yielding $n=5$. For trio interactions an expression similar to Eq. (1.2) holds to lowest order, with $d$ replaced by the perimeter of the triangle formed by the adatoms. Here the decay is even faster, with $n=7$. In short, interactions mediated by bulk states have negligible strength for values of $d$ for which the asymptotic expression is valid.

The situation is strikingly different when there is a metallic surface state (i.e. a surface band that cross the Fermi energy), such as found near the $\bar{\Gamma}$ point on the (111) faces of noble metals (associated with the (111) necks of the Fermi surface). For this case $n$ is 2 and $5 / 2$ for pair and trio interactions, respectively, so that the asymptotic regime is physically important [32]. Indeed, trio interactions may play a role in the formation of $2 \mathrm{D}$ clusters of $\mathrm{Cu} / \mathrm{Cu}(110)$ [33]. Furthermore, the Fermi "surface" is circular, and $k_{F}$ is much smaller than its bulk counterpart, leading to the dramatic oscillations (with wavelength $\pi / k_{F} \approx 15 \AA$ ) seen in STM experiments [34].

Most of the content of this and the second, third, and fifth sections of this paper were included in the first author's presentation at the $\mathrm{V}^{\text {th }}$ StranskiKaischew Surface Science Workshop (SK-SSW'2005): "Nanophenomena at Surfaces-Fundamentals of Exotic Condensed Matter Properties" in Pamporovo, but have been updated. Each section provides references from which the content was adapted and from which more information can be obtained. 


\subsection{Recollection of Two Effects on Statistical Mechanics}

In section we recall two remarkable implications of trio interactions for phase diagrams [16]. While these ideas are not new, they have been largely ignored by the community and so bear repeating.

\subsubsection{Pitfall in Transforming Trio Strength between Lattice Gas} and Ising Models

Many researchers recast the lattice gas model into an Ising model before doing computations. This seemingly innocent procedure invites pitfalls when multisite interactions are involved. Here we offer a particular example: what seems like a relatively modest 3 -spin interaction can correspond to an unphysically large trio interaction in the lattice gas Hamiltonian. Our example involves a square lattice with $\mathrm{M}=2$ (first and second neighbor pair interactions) and the right-triangle trio $E_{R T}$ corresponding to two $E_{1}$ legs at right angles and an $E_{2}$ hypotenuse (or $E_{112}$ for short, in a general formal notation [3])

The mapping to spin language, with $s_{i}= \pm 1$, is $n_{i}=\left(1+s_{i}\right) / 2$. We see

$$
\mathcal{H}=\left(\frac{E_{1}}{4}+\frac{E_{R T}}{2}\right) \sum_{\langle i j\rangle_{1}} s_{i} s_{j}+\left(\frac{E_{2}}{4}+\frac{E_{R T}}{4}\right) \sum_{\langle i j\rangle_{2}} s_{i} s_{j}+\frac{E_{R T}}{8} \sum_{\langle i j k\rangle_{R T}} s_{i} s_{j} s_{k}
$$

In Ising or spin language, the three coefficients are called $-J_{1},-J_{2}$, and $-J_{R T}$, respectively. For the pair interactions, we easily see that

$$
\frac{J_{2}}{J_{1}}=\frac{E_{2} / E_{1}+E_{R T} / E_{1}}{1+2 E_{R T} / E_{1}} \approx \frac{E_{2}}{E_{1}} \text { for }\left|E_{R T}\right| \lesssim 4\left|E_{1}\right| .
$$

One might then naively-but incorrectly-expect that $J_{R T} / J_{1} \approx E_{R T} / E_{1}$. Instead

$$
\frac{J_{R T}}{J_{1}}=\frac{E_{R T} / E_{1}}{2+4 E_{R T} / E_{1}} \Leftrightarrow \frac{E_{R T}}{E_{1}}=\frac{2 J_{R T} / J_{1}}{1-4 J_{R T} / J_{1}}
$$

This highly non-linear relation leads to a surprising result: For $J_{R T} / J_{1} \lesssim 1 / 4$, $E_{R T} / E_{1} \gg J_{R T} / J_{1}$. Furthermore, if $J_{R T} / J_{1}$ increases to slightly above $1 / 4$, $E_{R T} / E_{1}$ becomes large and negative. A similar effect would occur in the opposite direction for $E_{R T} / E_{1} \approx-1 / 2$, a larger magnitude than one is likely to encounter.

Finally, it is unfortunate (to say the least) that some researchers (including prominent ones) denote the lattice-gas energy parameters as $J$. Even in the simplest case of just a nearest neighbor interaction, the lattice gas energy differs from the Ising energy by a factor of 4 ; thus, misinterpretation and numerical inaccuracies are very likely.

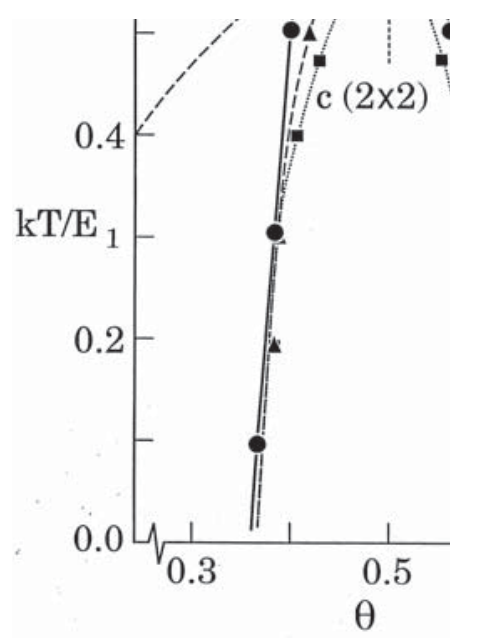

Fig. 1.2. Illustration, for a $\mathrm{c}(2 \times 2)$ overlayer on a square lattice, of the heuristic guideline, based on scaling the elementary excitation, used to predict which trios produce observable asymmetries in phase boundaries. For the simplest case of a NN repulsion, $E_{l}$, the disordering temperature is known exactly from the Onsager solution to the Ising model; it is indicated by the $\times$ on the temperature-coverage phase diagram. The effect of adding a right-triangle trio repulsion $E_{R T}$, a linear trio repulsion $E_{L T}$, or both was studied using Monte Carlo for the case $E_{R T}=E_{L T}=$ $E_{1} / 4$; the results for the three cases are plotted with filled triangles, squares, and circles, with dashed, dotted, and solid curves, respectively, added to guide the eve. The behavior at $\theta=0.5$ is anticipated by Eqs. (1.7) and (1.8). The dashed and dotted curves appear symmetric about $\theta=0.5$ (short-dotted line); only with both trios present does the [solid] phase boundary become noticeably asymmetric. In the plots at the right, the $\times$ 's and dots indicate occupied and vacant sites, respectively, in a perfectly ordered configuration, with the large symbols denoting a vacancy $(\theta<$ $1 / 2)$ or extra adatom $(\theta>1 / 2)$, respectively. The arrows on the plots depict the lowest-energy excitations, with the linetype of the shaft corresponding to the linetype of the phase boundary on the phase diagram; for each linetype, this excitation energy is the same for $\theta<1 / 2$ and $\theta>1 / 2$. According to the heuristic prescription, these energies scale the disordering temperature. When both trios are present, there is "frustration" over which excitation to use, the prescription fails, and asymmetries occur. In the phase diagram, the broad dashed curve is the result of a mean-field calculation, plotted at half its magnitude (i.e. $T_{c}(\theta=0.5) \approx 1.2 E_{l}$ ); not only is this prediction of $T_{c}$ far too high and too broad, it also erroneously predicts substantial asvmmetrv. From Ref. [16 


\subsubsection{Effect on Phase Boundaries: Asymmetries Not Inevitable}

A trio interaction, which breaks particle-hole symmetry (or up-down symmetry in the Ising viewpoint) is generally expected to lead inevitably to substantial asymmetries in phase diagrams about half-monolayer coverage. A rather surprising finding of numerical [Monte Carlo] calculations is that a single type of trio interaction need not necessarily do so. (See also the discussion by Persson of the role of adsorption in sites not of high symmetry, the equivalence to trio interactions, and the rich effects on temperature-coverage phase diagrams $[2,35]$.) This result, illustrated in Fig. 1.2 for the case of a c $(2 \times 2)$ overlayer with $E_{1}>0$ and $E_{R T}$, is in sharp contrast to the observation in 2D calculations which treat fluctuations approximately-mean field and quasi-chemical approximations - that trios must produce such asymmetries [36]. Likewise we see that a linear trio $E_{L T}$ alone does not produce an asymmetry. However, when both $E_{R T}$ and $E_{L T}$ are present, the expected notable asymmetry does appear. To comprehend this effect of trios on the phase boundary, we need some way to assess the difference in the way the trio interactions affect the lower-temperature ordered phase compared to the higher-temperature disordered phase. Evidently the breaking by trios of the particle-hole symmetry of the pair-interaction lattice-gas hamiltonian, and the resulting asymmetry in the ground state energy, is not the crux.

We describe a crude approximation scheme for assessing the change in the disordering temperature $T_{c}$ of an ordered phase from a known value $T_{c}(0)$ for some Hamiltonian to $T_{c}\left(E_{\text {new }}\right)$ for a more complicated Hamiltonian with a new interaction energy $E_{\text {new }}$. While we have applied our procedure [37] to a wide range of problems, we still have no formal derivation. In essence, the idea is that $T_{c}$ scales with the lowest-energy excitation from the ground state. In Ref. [37] we show, e.g., that for a $\mathrm{c}(2 \times 2)$ overlayer with half-monolayer coverage, characterized by a nearest-neighbor repulsion $E_{1}$ and a smaller secondneighbor interaction $E_{2}$,

$$
T_{c}\left(E_{2}\right)=T_{c}\left(E_{2}=0\right)\left(1-\frac{4 E_{2}}{3 E_{1}}\right)
$$

For this simple problem, Barber showed [38] that the exact coefficient is $\sqrt{ } 2 \approx 1.41$ rather than $4 / 3 \approx 1.33$; on the other hand, our value is much better than the mean-field prediction of 1 . For this same problem, the effect of a right-triangle trio interaction $E_{R T}$ (with $E_{2}=0$ ) is given by

$$
\frac{3 E_{1}+2 E_{R T}}{T_{c}\left(E_{R T}\right)}=\frac{3 E_{1}}{T_{c}(0)} \quad \Rightarrow \quad T_{c}\left(E_{R T}\right)=T_{c}\left(E_{R T}=0\right)\left(1+\frac{2 E_{R T}}{3 E_{1}}\right) .
$$

Similarly, for a linear trio $E_{L T}$

$$
\frac{3 E_{1}+E_{L T}}{T_{c}\left(E_{L T}\right)}=\frac{3 E_{1}}{T_{c}(0)} \quad \Rightarrow \quad T_{c}\left(E_{L T}\right)=T_{c}\left(E_{L T}=0\right)\left(1+\frac{E_{L T}}{3 E_{1}}\right)
$$

\section{T.L. Einstein and Rajesh Sathiyanarayanan}

We caution that this procedure is applicable only if the new interaction does not alter the symmetry of the ordered state and works well only if the nearby elementary excitation from the fully ordered state is uniquely defined. Thus, it works well for a $(\sqrt{ } 3 \times \sqrt{ } 3)$ overlayer on a hexagonal net but not for a $p(2 \times 2)$. It is also curious that this procedure requires a lattice gas picture in which the number of atoms is conserved (i.e. a canonical ensemble or Kawasaki dynamics); if the atom instead hopped to a "bath" (i.e. a grand canonical ensemble, or fixed chemical potential, or a single spin flip in a spin analogue (Glauber dynamics), the predictions are quite poor.

To assess the effect of trios on the symmetry of the temperature-coverage phase boundary of a $c(2 \times 2)$ overlayer, we look at the elementary excitation near a defect, either an extra adatom or a missing one. (See Fig. 1.2.) For just a right-triangle trio, there are no such trios (no $2 E_{R T}$ ) in the excited state when there is a vacancy; when there is an extra, there are two RT trios in the ordered state which are lost in hopping to the nearest neighbor (where another two RT trios occur). So in both cases, there is no change in the number of RT trios, i.e. no change proportional to $E_{R T}$ is involved. A similar effect occurs with a linear trio, but with a different elementary excitation. (See Fig. 1.2.) In either case, we saw that the phase boundary computed using Monte Carlo appears symmetric. Only when both trios are present does a marked asymmetry occur. However, a noteworthy inadequacy of this simple picture is its inability to give any idea of the coverage-dependence of $T_{c}$ [39].

\subsection{Applications to Gases on Metals}

As an example of the state of the art in quantitatively determining adsorbateadsorbate interactions from combined theory and experiment, we discuss the study of $\mathrm{c}(2 \times 2) \mathrm{N}$ on $\mathrm{Fe}(001)$ by Österlund et al. [8]. Since there is just one ordered phase, one might expect to be able to extract information about only a handful of interactions, say $E_{1}>0, E_{2}$, and a trio, from STM observations. The authors use a new concept called configuration distribution analysis (CDA) to extract much more information from high-resolution images. Around each nitrogen adatom they obtain a site map of possible adsorption sites. By comparing the resulting experimental conditional probabilities with those obtained from Monte Carlo simulations with a lattice-gas model, they can refine estimates of the various interactions.

The authors first consider a long-established analysis [40,41] of the pair correlation function $g(j)$, from which they deduce $E_{j}=-k_{B} T \ln g(j)$, for $j$ th neighbors, between fractional coverages $\Theta=0.037$ and 0.15 . They find very little NN site occupation and enhanced NNN occupation. For $j>4$ there is no significant deviation from a random distribution $(g=1)$. From the data they find $E_{1}=0.13 \mathrm{eV}$ and $E_{2}=-0.013 \mathrm{eV}$. Concerned about poor statistics at $\Theta=0.037$, the authors also carried out Monte Carlo simulations and used a least-squares fit of data at all the coverages, finding similar energies: $E_{1}=$ 
$0.14 \mathrm{eV}, E_{2}=-0.023 \mathrm{eV}$, and $E_{3}=0.003 \mathrm{eV}$. They then did a CDA analysis in conjunction with Monte Carlo simulations (seemingly at $\Theta=0.108$ ) supplemented by a comparison of measured and simulated island-size distributions. They thus determined $E_{1}=0.13 \mathrm{eV}, E_{2}=-0.018 \mathrm{eV}, E_{3}=0.019 \mathrm{eV}$, and trio interaction energies $E_{223}=-0.012 \mathrm{eV}$, and $E_{225}=0.006 \mathrm{eV}$. (These trios correspond to RT and LT triangles on a larger scale, with insignificant direct interactions.) If $3 \mathrm{NN}$ (third $\mathrm{NN}$ ) sites are considered in the CDA, then $E_{3}$ decreases to $0.015 \mathrm{eV}$. If trios are omitted from the CDA analysis, $E_{1}$ is unchanged but $E_{2}$ increases in magnitude to $-0.038 \mathrm{eV}$, while $E_{3}$ diminishes to $0.003 \mathrm{eV}$. In other words, fitted pair interactions are actually effective interactions that include omitted interactions in some average, unspecified way. The effect seems to be largest for the most prominent legs of the omitted interactions, in this case NNN.

The authors also include an elastic repulsion, taken to have the asymptotic isotropic form $C / R^{3}$ with strength $C / a^{3}=0.15 \mathrm{eV}$, where $a$ is the Fe lattice constant $(2.87 \AA)$. Hence, the electronic components of the 3 pair interactions (for the CDA analysis including trios and configurations to $j=3$ ) are $-0.2 \mathrm{eV}$, $-0.71 \mathrm{eV}$, and $-0.04 \mathrm{eV}$, respectively. (There is no comment about adjusting the trios.) Note, remarkably, that $\left|E_{1}^{e l}\right|<\left|E_{2}^{e l}\right|$. The authors conclude that the electronic interaction is short range. The embedded atom method (EAM), for metals on metals, predicts that the indirect interaction is repulsive and proportional to the number of shared substrate atoms [3]. On very general grounds one expects $R^{-5}$ decay of the envelope in Eq. (1.2). We caution that the distinction between electronic and elastic has long been recognized as subtle [42] and that the asymptotic limit of the elastic interaction is likely to be significantly modified at separations $\mathcal{O}(a)$ by more rapidly decaying terms in the multipole series series. [43]

\subsection{Refined Schemes for Extracting Interaction Energies}

To extract estimates of interaction energies when there are many such parameters is a delicate task. Typically one obtains a large number of simultaneous equations by computing the total interaction of a large variety of different overlayer structures. One should have more overlayer structures than interactions so as to be able to check for robustness of the deduced values. While informal schemes had formerly seemed sufficient [44], it is safer and sounder to use formal cross-validation schemes used by several groups $[13,45]$ to study overlayer systems.

In recent work [46] we used the leave- $n_{v}$-out cross-validation method [47] to fit the computed energies for $\mathrm{Cu}(110)$ to the interaction parameters (cf. Section 1.6). This method is expected to perform better than the more-commonlyused leave-1-out cross-validation scheme [47]. We calculated the interaction strengths in the following way: for a particular supercell, we computed total energies for, say, $n$ different configurations of adatoms. In addition, we posit

\section{T.L. Einstein and Rajesh Sathiyanarayanan}

the number of significant interactions $n_{i}$. We then use $n_{i}$ (out of $n$ ) equations to solve for the interaction energies. These interactions are then used to predict the energies of the remaining $n_{v}=n-n_{i}$ equations. We then compute the root mean squared (rms) of the prediction error per adatom for all configurations $j\left(1 \leq j \leq n_{v}\right)$, each with $a_{j}$ adatoms in it:

$$
\Delta E_{\mathrm{rms}}=\sqrt{\frac{1}{n_{v}} \sum_{j=1}^{n_{v}}\left(\Delta E_{j}\right)^{2}} \quad \Delta E_{j}=\frac{E^{\mathrm{pred}}-E^{\mathrm{VASP}}}{a_{j}}
$$

We repeat this procedure for different partitions of $\left(n, n_{i}\right)$, and sets of interactions from only those partitions whose $\Delta E_{\text {rms }}$ values are lower than a specified threshold value $(10 \mathrm{meV}$ /adatom in our case) are considered for the final averaging of interaction values. Finally, we find the value of $n_{i}$ that leads to the best convergence. As a check, we perform this procedure on two different computational supercells.

\subsection{Effect of Relaxations in Homoepitaxy with Direct Lateral Interactions}

When direct interactions play a significant role, such as for $(1 \times 1)$ homoepitaxial partial monolayers, one must be wary of relaxation-induced modification of energies near steps or island edges. From bond-energy, bond-order, bond-length reasoning [48], one can expect such atoms - which lack their full complement of near-neighbor bonds, to move closer to the remaining neighbors to partially compensate for the loss. Persson showed that relaxation in the form adsorption away from high-symmetry sites can lead to effective trio interactions, and attendant effects, in systems with ostensibly only pair interactions $[2,35]$. Our goal here is to show that these relaxation effects are especially significant for multi-site interactions, where the relaxations are not along the bond directions. Furthermore, multi-site interactions, in general, have a large elastic component; hence, a careful consideration of relaxation effects is needed while computing them. We discuss in particular how strain related effects are important when calculating the step stiffness on $\mathrm{Cu}(100)$. Because of adatom relaxation near steps, the inclusion of non-pairwise, quarto interaction between four adatoms is required on this square-lattice surface in order to preserve a lattice-gas description.

\subsubsection{Multisite Interactions in Step-Stiffness Asymmetry}

Step stiffness (which earlier served as the mass in the 1D fermion model of steps) underlies how steps respond. It is one of the three parameters of the step-continuum model, [49] which has proved a powerful way to describe step behavior on a coarse-grained level, without recourse to a myriad of microscopic 
energies and rates. In the analogy between $2 \mathrm{D}$ configurations of steps and worldlines of spinless fermions in $(1+1) \mathrm{D}$, step stiffness $\tilde{\beta}$ plays the role of the mass of the fermion. As the inertial term, stiffness determines how a step responds to fluctuations, to driving forces, and to interactions with other steps.

We summarize our lattice-gas-based computations of the orientation dependence of step stiffness for the (001) and (111) faces of $\mathrm{Cu}[50,51]$. This work illustrates both successes and some shortcomings of using a lattice-gas model with just NN interactions: whereas the step stiffness on $\mathrm{Cu}(111)$ is well described by $\mathrm{NN}$ interactions alone, the step stiffness on $\mathrm{Cu}(001)$ requires the inclusion of NNN and perhaps even trio interactions. We discuss only the latter.

The step stiffness $\tilde{\beta}(\theta) \equiv \beta(\theta)+\beta^{\prime \prime}(\theta)$ weights deviations from straightness in the step Hamiltonian, where $\theta$ is conventional designation of the azimuthal misorientation angle; it measured from the close-packed direction. Here $\beta$ is the step free energy per length (or, equivalently, the line tension, since the surface is maintained at constant [zero] charge [52]). The stiffness is inversely proportional to the step diffusivity, which measures the degree of wandering of a step perpendicular to its mean direction. This diffusivity can be readily written down in terms of the energies $\varepsilon_{k}$ of kinks along steps with a mean orientation $\theta=0$ : in this case, all kinks are thermally excited. Conversely, experimental measurements of the low-temperature diffusivity (via the scale factor of the spatial correlation function) can be used to deduce the kink energy. A more subtle question is how this stiffness depends on $\theta$. Even for temperatures much below $\varepsilon_{k}$, there are always a non-vanishing number of kinks when $\theta \neq 0$, the density of which are fixed by geometry (and so are proportional to $\tan \theta$ ). In a bond-counting model, the energetic portion of the $\beta(\theta)$ is canceled by its second derivative with respect to $\theta$. so that the stiffness is due to the entropy contribution alone. Away from close-packed directions, this entropy can be determined by simple combinatoric factors at low temperature $T$. [53-55]

Interest in this whole issue has been piqued by Dieluweit et al.'s finding [56] that the stiffness as predicted in the above fashion, assuming that only NN interactions $E_{1}$ are important, underestimates the values for $\mathrm{Cu}(001)$ derived from two independent types of experiments: direct measurement of the diffusivity on vicinal $\mathrm{Cu}$ surfaces with various tilts and examination of the shape of (single-layer) islands. The agreement of the two types of measurements assures that the underestimate is not an anomaly due to step-step interactions. In that work, the effect of NNN interactions $E_{2}$ was crudely estimated by examining a general formula obtained by Akutsu and Akutsu, [57] showing a correction of order $\exp \left(-E_{2} / k_{B} T\right)$, which was glibly deemed to be insignificant. In subsequent work the Twente group [58] considered steps in just the two principal directions and showed that by including an attractive NNN interaction, one could evaluate the step free energies and obtain a ratio consistent with the

\section{T.L. Einstein and Rajesh Sathiyanarayanan}

experimental results in Ref. [56]. They later extended their calculations [59] to examine the stiffness.

To make contact with experiment, one typically first gauges the diffusivity along a close-packed direction and from it extracts the ratio of the elementary kink energy $\varepsilon_{k}$ to $T$. Arguably the least ambiguous way to relate $\varepsilon_{k}$ to bonds in a lattice gas model is to extract an atom from the edge and place it alongside the step well away from the new unit indentation, thereby creating four kinks. [60] Removing the step atom costs energy $3 E_{1}+2 E_{2}$ while its replacement next to the step recovers $E_{1}+2 E_{2}$. Thus, whether or not there are NNN interactions, we identify $\varepsilon_{k}=-\frac{1}{2} E_{1}=\frac{1}{2}\left|E_{1}\right|$ (since the formation of $\mathrm{Cu}$ islands implies $\left.E_{1}<0\right)$; thus, as necessary, $\varepsilon_{k}>0$.

In the low-temperature limit, appropriate to the experiments [56], we have shown that

$$
\frac{k_{B} T}{\tilde{\beta} a}=\frac{m \sqrt{(1-m)^{2}+4 m e^{E_{2} / k_{B} T}}}{\left(1+m^{2}\right)^{3 / 2}} \underset{m \rightarrow 0^{+}}{\longrightarrow} \frac{m+m^{2}}{\left(1+m^{2}\right)^{3 / 2}}
$$

where $m$ is the step-edge in-plane slope.

Fig. 1.3 compares Eq. (1.10) to corresponding exact solutions at several temperatures when $E_{2}=E_{1} / 10$. We see that Eq. (1.10) overlaps the exact solution at temperatures as high as $T_{c} / 6$. As the temperature increases, the stiffness becomes more isotropic, and Eq. (1.10) begins to overestimate the stiffness near $\theta=0^{\circ}$. In Fig. 1.4 (using the experimental value [61] $\varepsilon_{k}=128$ $\mathrm{meV} \Rightarrow E_{1}=-256 \mathrm{meV}$ ), we compare Eq. (1.10) to the NN-Ising model at $T=320 \mathrm{~K}$, as well as to the experimental results of Ref. [56]. For strongly attractive (negative) $E_{2}, k_{B} T / \tilde{\beta} a$ decreases significantly. In fact, when $E_{2} / E_{1}$ is $1 / 6$, so that $-E_{2} / 2 k_{B} T=\left(E_{2} / E_{1}\right)\left(\varepsilon_{k} / k_{B} T\right) \approx(1 / 6) 4.64$, the modelpredicted value of $k_{B} T / \tilde{\beta} a$ has decreased to less than half its $E_{2}=0$ value, so about $3 / 2$ the experimental ratio. For the NNN interaction alone to account

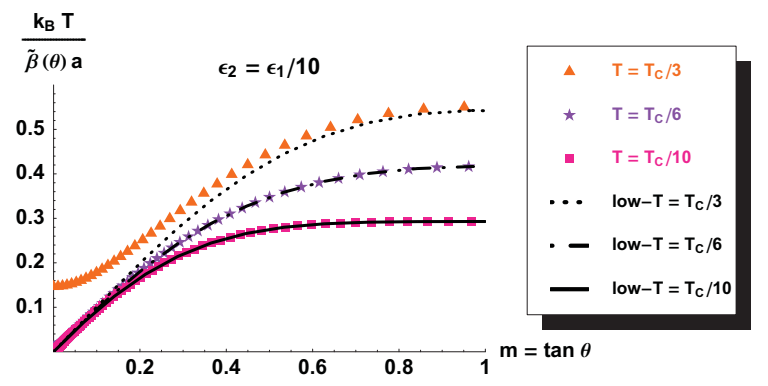

Fig. 1.3. The range of validity of Eq. (1.10) is examined by comparing it to exact numerical solutions of the SOS model at several temperatures. In the legend $T_{c}$ refers to the NN lattice-gas (Ising) model; for $\left|E_{1}\right|=256 \mathrm{meV}, T_{c}=1685 \mathrm{~K}$. From Ref. [50]. 
for the factor-of-4 discrepancy between model/theory and experiment [56], Fig. 1.4 shows that $E_{2} / E_{1} \approx 0.3$ would be required.

\subsubsection{Effect of Trio Interactions}

If we include a RT trio $\left(E_{112}\right)$ the effective NN lattice-gas energy $E_{1}^{\text {eff }}$ is $E_{1}+2 E_{R T}$ and, more significantly the effective NNN interaction energy is $E_{2}+E_{R T}$. Thus, $E_{R T}$ must be attractive (negative) if it is to help account for the discrepancy in Fig. 2 of Ref. [56] between the model and experiment. Furthermore, by revisiting the configurations discussed in the penultimate paragraph of the Introduction, we find that the kink energy $\varepsilon_{k}$ becomes $-\frac{1}{2} E_{1}-E_{R T}$. Thus, for a repulsive $E_{R T},\left|E_{1}\right|$ will be larger than predicted by an analysis of, e.g., step-edge diffusivity that neglects $E_{R T}$. Lastly, the closepacked edge energy, i.e. the $T=0$ line tension $\beta(0)=-\frac{1}{2} E_{1}-E_{2}$, becomes $-\frac{1}{2} E_{1}-E_{2}-2 E_{R T}$

We also investigated the strain related effects on calculated trio interaction energies on $\mathrm{Cu}(100)$, where Dieluweit et al. [56] showed that the NN Ising model cannot explain the experimentally observed step-stiffness anisotropy. In a response, we showed that the addition of an effective NNN attractive interaction could resolve the discrepancy. The effective NNN interaction $\left(E_{2}\right)$ can be written as the sum of two components as illustrated in Fig. 1.1a:

- a pairwise second neighbor interaction energy $\left(E_{2}\right)$

- an orientation-dependent right-isosceles trio interaction energy $\left(E_{R T}\right)$

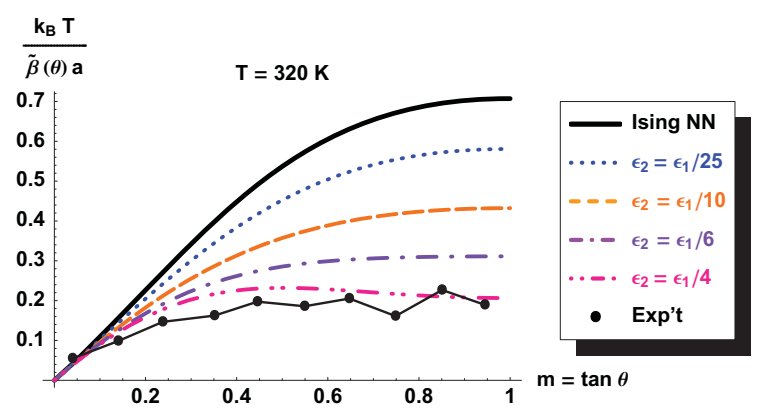

Fig. 1.4. Eq. (1.10) is plotted for a range of values of $E_{2}$ (epsilon on the plot, where $E_{1}$ and $E_{2}$ are NN- and NNN-interaction energies, respectively, in a lattice-gas picture. The solid curve denoted "Ising NN" corresponds to $E_{2}=0$. The dots labeled "Exp't" are taken from Fig. 2 of Ref. [56] and were derived from the equilibrium shape of islands on $\mathrm{Cu}(001)$ at $302 \mathrm{~K}$, with line segments to guide the eye. To clarity, we omit similar data derived from correlation functions of vicinal surfaces at various temperatures. Note that for $E_{2}=E_{1} / 4$ there is a maximum near $\tan \theta=1 / 2$ that is not observed in experimental data. From Ref. [50].
14 T.L. Einstein and Rajesh Sathiyanarayanan

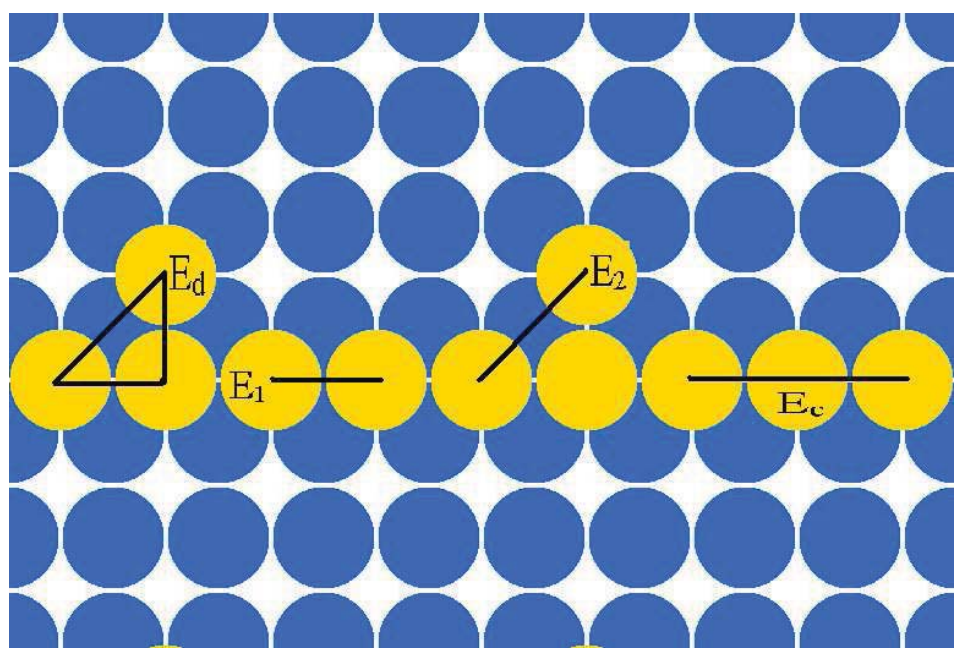

Fig. 1.5. (a)Effective NNN interactions on a (100) surface [of an fcc crystal], (b) Multisite interactions $E_{d}$ (solid triangle), $E_{d}^{\prime}$ (broken triangle) and $E_{Q}$ (square), where the subscript d is RT in our notation. The trio $E_{d}$ has adatoms on the step edge whereas $E_{d}^{\prime}$ has no adatoms on the step edge. From Ref. [24]

However, when we used VASP to calculate these interactions, we found a large repulsive value of around $50 \mathrm{meV}$ for the right-isosceles trio interaction energy $\left(E_{R T}\right)$. Such a value cancels the calculated attractive second-neighbor interactions $\left(E_{2}\right)$ thereby reducing the model to a NN Ising model. Thus, in the end the discrepancy between theory and experiment could not be resolved.

To see the effect of relaxation, we repeated the calculations with a bigger supercell $(4 \mathrm{x} 4 \mathrm{x} 14)$. If an adatom stripe has kinks, there can be two types of right-isoceles trios, ones with one/two adatoms on the step-edge and ones with no adatoms on the step-edge (Fig. 1.1b). Since the local geometry of these two adatoms is different, we could expect the trio interaction energies to be different because the RT trio adatoms $\left(E_{R T}^{\prime}\right)$ inside a stripe cannot relax as much laterally as the trios with its vertices on the step $\left(E_{R T}\right)$. The trio energy that we calculated earlier corresponds to a linear combination of $E_{R T}$ and $E_{R T}^{\prime}$, weighted more dominantly by $E_{R T}^{\prime}$. However, the calculation of the step stiffness depends on broken step-edge trios, which necessarily correspond to $E_{R T}$. To distinguish these two trios here, we calculated the energies of four different adatom configurations and we solved the resultant linear system of equations. With this correction, at the step-edge $E_{R T} \approx 12.5 \mathrm{meV} /$ atom, and the effective NNN interaction is thus $E_{2}^{\text {eff }}=E_{2}+E_{R T} \approx-35 \mathrm{meV} /$ atom

Though $E_{R T}$ is still repulsive, its magnitude lower than that of the attractive $E_{2}$. The ratio of the effective NN interaction to the effective NNN 


$$
E_{2}^{\mathrm{eff}} / E_{1}^{\mathrm{eff}} \sim 1 / 9 \quad \text { while } \quad E_{2} / E_{1} \sim 1 / 7
$$

which is much closer to experimental expectations.

Distinguishing between the step-edge trios $E_{R T}$ and the bulk trios $E_{R T}^{\prime}$ is not compatible with a proper lattice-gas picture, where interactions should not depend on local position and geometry. We can remedy this problem by introducing a quarto interaction. This quarto interaction distinguishes between the two trios because is present only in bulk trios $E_{R T}^{\prime}$ :

$$
E_{R T}^{\prime}=(3 / 4) E_{Q}+E_{R T}
$$

This yields the value of the quarto interaction to be $E_{Q}=53 \mathrm{meV}$. This is a significant amount of energy (compared with collinear trio $E_{L T}=-15$ $\mathrm{meV} /$ atom and third-nearest neighbor interaction $E_{3}=-8 \mathrm{meV} /$ atom [62]) and hence is likely to have consequences in calculations of other properties.

In summary, when calculating trio interactions from first principles, however, care must be taken; they can be exquisitely sensitive to the geometry and structure of the supercell used to calculate them. Such sensitivity to local relaxation can complicate a simple lattice-gas description. To account for the relaxation of trios near step edges, for example, we introduce a non-pairwise quarto interaction $E_{Q}$ among four neighboring adatoms. We find that such an interaction is necessary to bridge the theoretical step stiffness with experimental measurements on $\mathrm{Cu}(100)$. In that case, we find what amounts to a relatively large, repulsive quarto interaction $E_{Q}=54 \mathrm{meV}$ that has significant physical consequences in our problem and presumably more generally. (In the same paper [24] we find that the inclusion of trio interactions can account for the difference in A- and B-step formation energies on $\mathrm{Pt}(111)$.)

\subsection{Connector Models}

The expansion in $m$-adatom interactions can become cumbersome and unwieldy if the multiparticle energies do not become smaller after, say, quarto interactions. Seeking an alternative approach to circumvent this issue, Tiwary and Fichthorn (TF) [25] proposed the connector model (named after the construction-kit toy [63]), which focuses on the vertices rather than the links of a cluster of adatoms. For example, consider 4 adatoms forming a NN square. In the lattice-gas model, the total energy would be $4 E_{1}+2 E_{2}$ $+4 E_{R T}+E_{Q}$. In the connector model with just NN spokes (spokes of half the NN bond length and oriented in the appropriate directions), this total energy is just 4 times the energy of a connector hub with two perpendicular spokes. For this lattice, there are just 5 types of connectors, having 1, 2, 3, or 4 spokes, the 2 -spoke case having a straight and a right-angle conformation. More often, one must use connectors with NN and NNN spokes. For the square of 4 adatoms, there would still be just one connector, with a NNN

\section{T.L. Einstein and Rajesh Sathiyanarayanan}

spoke between the two perpendicular NN spokes (called D6 in Ref. [25]). For the straight-conformation 2-NN-spoke case, one can add 1 NNN spoke in 1 distinct conformation, 2 NNN spokes in 3 conformations, 3 or 4 (or 0) each in 1 distinct conformation, summing to 7 possibilities. In other cases there will be even more. With $3 \mathrm{NN}$ spokes, the number of possibilities is even larger. One of the main features of this model is that the type of connector contains information about the local geometry of the adatom; hence relaxation effects are expected to be built into the model. However, to keep the number of fitted energies reasonable, TF use just 8 connectors: D1 for an isolated atom; D2 and D3 for just 1 NN or NNN spoke, respectively; D4 and D5 for 2 NN or NNN spokes in the same direction (linear), respectively; D7 with 3 NN spokes and 2 NNN spokes between them; and D 8 for all 8 spokes. Thus, as for lattice gas interactions, success requires that the investigator have enough insight to select the connectors that capture the essence of the problem.

Other applications to date have been to (110) fcc surfaces, where the rectangular symmetry leads to a large variety of lattice-gas energies as well as a much greater number of connectors, since the perpendicular "NN" spokes have different lengths (though NNN are still the same). However, the multiplicity can be reduced by sometimes neglecting some differences. We used ten connectors (shown in Fig. 1.6) to characterize adatom interactions on $\mathrm{Cu}(110)$ [46], while $\mathrm{TF}$ used the first 9 for $\mathrm{Al}(110)$.

For $\mathrm{Cu}(110)$ the $\mathrm{CV}$ scores are as good as those obtained using a dozen lateral interactions in the lattice-gas approach [46]. This agreement is plausible since it is often possible to establish linear relations between the connector and lattice-gas energies, e.g.

$$
C_{6}=E_{0}+\frac{E_{1}}{2}+\frac{E_{2}}{2}+\frac{E_{3}}{2}+\frac{E_{T 1}}{3}+\frac{E_{Q 1}}{4}
$$

The sensitivity of multi-site interactions to relaxation is not apparent from the connector energy values because (i) each connector has contributions from adsorption energy $\left(E_{0}\right.$ or $\left.C_{1}\right)$ and other pairwise interactions that dominate over contributions from multi-site interactions; also, the contribution from a particular multi-site interaction is divided by the number of participating adatoms (cf. Eq. (1.13), further decreasing the sensitivity of connector energies to adatom relaxations. However, the connector model incorporates such relaxation effects, as can be seen from the uniformly low CV scores for all relaxation schemes [46]. Evidently the connector model works well in the cases of $\mathrm{Cu}(110), \mathrm{Al}(110)$, and $\mathrm{Al}(100)$. It remains to be seen whether the connector model provides an adequate solution, without the need for any ad hoc patches, to the overlayer problem. Relaxation effects become prominent during energy calculations of adatoms near step edges; in such calculations the simple lattice-gas model encounters problems $[24,62]$. At the same time, accommodating the relaxation effects encountered in such calculations within the connector model might require the usage of connectors that account for the orientations of neighbor bonds, resulting in an undesirably large num- 


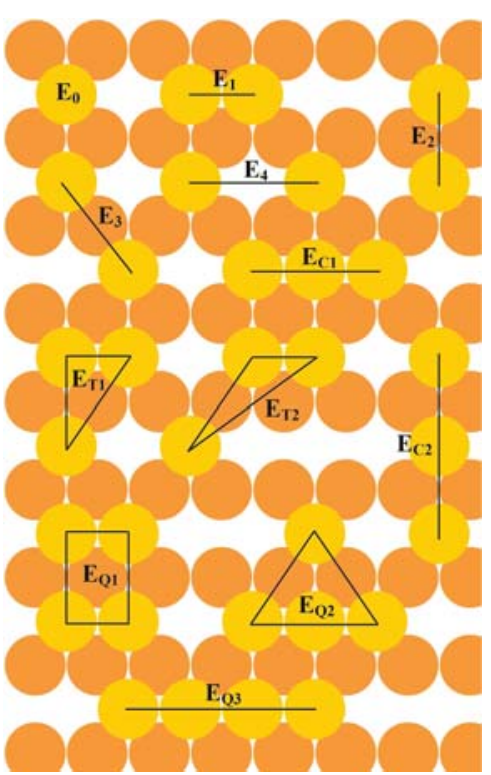

Fig. 1.6. Left: Connectors [25] used to characterize $\mathrm{Cu}$ adatom interactions on $\mathrm{Cu}(110)$. Lighter (mustard) circles represent adatoms and darker (orange) circles represent atoms in the substrate layer. Right: Lattice-gas interactions used to characterize $\mathrm{Cu}$ adatom interactions on $\mathrm{Cu}(110)$. Multi-site interactions $E_{T 2}, E_{Q 2}$ and $E_{Q 3}$ were found to be insignificant. From Ref. [46], which also provides tables of the values of these energies.

ber of connectors in the model. A DFT-based study that compares these two models on a surface like Pt(111), where such lateral relaxation is known to complicate surface energy calculations [24], might elucidate this issue.

\subsection{Interactions between Organic Molecules}

Understanding the interactions between and consequent self-assembly of organic molecules on metal surfaces has drawn much recent interest. The adsorption bond is of order an order of magnitude weaker than for the chemisorbed systems considered above. Direct interactions between the organic molecules are often by way of hydrogen bonds, and there is always a van der Waals attraction which, for these systems, may play the dominant role. While the coupling to the substrate is relatively weak, the indirect electronic interactions between adsorbates may be important at long-range when the substrate has metallic surface states
Very recently we have considered the adsorption of benzene on $\mathrm{Cu}(111)$ [64], using a DFT approach with a van der Waals functional included. We can account for the two ordered submonolayer phases observed by Dougherty et al. [65]. The denser phase is due to direct van der Waals bonding between the benzenes while the less dense phase appears to be due to the surfacestate-mediated interaction. We have not yet investigated the role of trio interactions in this system. Our ultimate goal is to explain the dramatic giant regular honeycomb structure formed by anthraquinone (AQ) molecules on this substrate [66]. In models that treat the admolecules as single "atoms", a repulsive trio interaction is crucial to prevent the formation of dense, unphysical overlayer regions [67]. Our belief is that the large, regular structure is related to interactions between $\mathrm{AQ}$ mediated by the metallic surface state. A variety of theoretical and experimental techniques are in progress to confirm this picture [68].

\subsection{Acknowledgments}

Our research at Maryland was supported primarily by the NSF-MRSEC, Grant DMR 05-20471. Some aspects benefited from NSF Grant Chem 0750334 and DOE-CMSN support. The Center for Nanophysics and Advanced Materials (CNAM) provided ancillary support. We thank T.J. Stasevich, P. Hyldgaard, K. Berland, E.D. Williams, L. Bartels, and K. Kim for fruitful collaboration and stimulating discussions on the topics discussed.

\section{References}

1. L. D. Roelofs, in Chemistry and Physics of Solid Surfaces, IV, edited by R. Vanselow and R. Howe (Springer-Verlag, Berlin, 1982), 219

2. B.N.J. Persson, Surface Sci. Rept. 15, 4 (1992).

3. T.L. Einstein, in Physical Structure of Solid Surfaces, edited by W.N. Unertl (Elsevier, Amsterdam, 1996).

4. A. Patrykiejew, S. Sokolowski, and K. Binder, Surf. Sci. Rept. 37, 207 (2000).

5. D.P. Landau, S.P. Lewis, and H.-B. Schüttler, editors, Computer Simulation Studies in Condensed-Matter Physics, vols. 1-19 (Springer Proceedings in Physics, Berlin, 1988-2008).

6. P.A. Rikvold, G. Brown, M.A. Novotny, and A. Wieckowski, Colloids and Surfaces A: Physicochemical and Engineering Aspects 134, 3 (1998).

7. L.D. Roelofs, in Physical Structure of Solid Surfaces, edited by W.N. Unertl (Elsevier, Amsterdam, 1996)

8. L. Österlund, M. Ø. Pedersen, I. Stensgaard, E. Lægsgaard, and F. Besenbacher, Phys. Rev. Lett. 83, 4812 (1999)

9. A. Bogicevic, S. Ovesson, P. Hyldgaard, B. I. Lundqvist, H. Brune, and D. R. Jennison, Phys. Rev. Lett. 85, 1910 (2000)

10. J.-S. McEwen, S. H. Payne, and C. Stampfl, Chem. Phys. Lett. 361, 317 (2002). 
11. W. Luo and K. A. Fichthorn, Phys. Rev. B 72, 115433 (2005).

12. A. P. J. Jansen and W. K. Offermans, Lec. Notes Comp. Sci. 3480 , 1020 (2005)

13. C. Stampfl, Catalysis Today 105, 17 (2005); M. Borg et al., Chem. Phys. Chem. 6, $1923(2005)$.

14. G. Kresse and J. Hafner, Phys. Rev. B 47, R558 (1993); 49, 14251 (1994).

15. G. Kresse and J. Furthmüller, Comput. Mater. Sci. 6, 15 (1996); Phys. Rev. B 54, 11169 (1996).

16. T.L. Einstein, Langmuir 7, 2520 (1991).

17. G. Ehrlich and F. Watanabe, Langmuir 7, 2555 (1991).

18. S.-J. Koh and G. Ehrlich, Phys. Rev. B 60, 5981 (1999).

19. Y. Zhang, V. Blum, K. Reuter, Phys. Rev. B 75, 235406 (2007).

20. Y. Tiwary, K.A. Fichthorn, Phys. Rev. B 75, 235451 (2007).

21. C. Lazo and F. J. Keil, Phys. Rev. B 79, 245418 (2009).

22. W.A. Harrison, Phys. Rev. B 7, 2408 (1973).

23. L.D. Roelofs, S.M. Foiles, M.S. Daw, and M.I. Baskes, Surf. Sci. 234, 63 (1990).

24. Rajesh Sathiyanarayanan, T.J. Stasevich, and T.L. Einstein, Surface Sci. 602, 1243 (2008).

25. Y. Tiwary and K. A. Fichthorn, Phys. Rev. B. 78, 205418 (2008).

26. T.L. Einstein, in Chemistry and Physics of Solid Surfaces II, edited by R Vanselow (CRC Press, Boca Raton, FL, 1979).

27. P. J. Feibelman, Annual Rev. Phys. Chem. 40, 261 (1989)

28. J.K. Nørskov, in The Chemistry and Physics of Solid Surfaces, vol. 6, edited by D.A. King and D.P. Woodruff (Elsevier, Amsterdam, 1993).

29. S. P. Timoshenko and J. N. Goodier, Theory of Elasticity, 3rd ed. (McGraw-Hill, New York, 1970).

30. T.L. Einstein and J.R. Schrieffer, Phys. Rev. B 7, 3629 (1973).

31. C. Kittel, in Solid State Physics, edited by F. Seitz, D. Turnbull, and H. Ehrenreich (Academic, New York, 1968), vol. 22, p. 1, and references therein.

32. P. Hyldgaard and M. Persson, J. Phys.: Condens. Matter 12, L13 (2000); P. Hyldgaard and T. L. Einstein, Europhysics Letters 59, 265 (2002).

33. P. Hyldgaard and T. L. Einstein, Surf. Sci. 532-535, 600 (2003); J. Cryst. Growth 275, e1637 (2005).

34. E.g., M.F. Crommie, C.P. Lutz and D.M. Eigler, Science 262, 218 (1993).

35. B.N.J. Persson, Surf. Sci. 258, 451 (1991).

36. A. Milchev, J. Chem. Phys 78, 1994 (1983); Electrochem. Acta 28, 941 (1983); A. Milchev and M. Paunov, Surface Sci. 108, 25 (1981)

37. N.C. Bartelt, T.L. Einstein, and E.D. Williams, J. Vac. Sci. Technol. A 2, 1006 (1984)

38. M.N. Barber, J. Phys. A 15, 915 (1982).

39. Michael E. Fisher, private communication.

40. T.T. Tsong and R. Casanova. Phys. Rev. Lett. 47, 113 (1981); T.T. Tsong, Mat. Sci. Engin. A 353, 1 (2003).

41. F. Watanabe and G. Ehrlich, J. Chem. Phys. 96, 3191 (1992)

42. Robert B. Griffiths, comment during discussion, Conf. on Phase Transitions on Surfaces, Orono, ME, Aug. 1981)

43. Cf., for steps, R. Najafabadi, D. J. Srolovitz, Surf. Sci. 317, 221 (1994).

44. T. J. Stasevich, T.L. Einstein, and S. Stolbov, Phys. Rev. B 73, 115426 (2006)

45. T. Mueller and G. Ceder, Phys. Rev. B 80, 024103 (2009), use cross validation in the context of a Bayesian approach to cluster expansions.
46. Rajesh Sathiyanarayanan and T.L. Einstein, , Surface Sci. 603, 2387 (2009). 47. J. Shao, J. Am. Stat. Assoc. 88, 486 (1993).

48. H. S. Johnston and C. A. Parr, J. Am. Chem. Soc. 85, 2544 (1963); W.H Weinberg and R.P. Merrill, Surface Sci. 33, 493 (1972); C. Q. Sun, B. K. Tay, X. T. Zeng, S. Li, T. P. Chen, J. Zhou, H. L. Bai, and E. Y. Jiang, J. Phys.: Condens. Matter 14, 7781 (2002).

49. H.-C. Jeong and E. D. Williams, Surf. Sci. Rept. 34, 171 (1999).

50. T. J. Stasevich, T. L. Einstein, R. K. P. Zia, M. Giesen, H. Ibach, and F. Szalma, Phys. Rev. B 70, 245404 (2004).

51. T. J. Stasevich, H. Gebremariam, T. L. Einstein, M. Giesen, C. Steimer, and H. Ibach, Phys. Rev. B 71, 245414 (2005) [cond-mat/0412002].

52. This simple equivalency does not hold for stepped surfaces in an electrochemical system, where the electrode potential $\phi$ is fixed rather than the surface charge density conjugate to $\phi$. H. Ibach and W. Schmickler, Phys. Rev. Lett. 91, 016106 (2003).

53. C. Rottman and M. Wortis, Phys. Rev. B 24, 6274 (1981).

54. J.E. Avron, H. van Beijeren, L. S. Schulman, and R. K. P. Zia, J. Phys. A 15 L81 (1982); R.K.P. Zia and J.E. Avron, Phys. Rev. B 25, 2042 (1982).

55. J.W. Cahn and R. Kikuchi, J. Phys. Chem. Solids 20, 94 (1961).

56. S. Dieluweit, H. Ibach, M. Giesen, and T. L. Einstein, Phys. Rev. B 67, 121410(R) (2003).

57. N. Akutsu and Y. Akutsu, Surf. Sci. 376, 92 (1997).

58. R. Van Moere, H. J. W. Zandvliet, and B. Poelsema, Phys. Rev. B 67, 193407 (2003).

59. H. J. W. Zandvliet, R. Van Moere, and B. Poelsema, Phys. Rev. B 68, 073404 (2003).

60. R. C. Nelson, T. L. Einstein, S. V. Khare, and P. J. Rous, Surf. Sci. 295, 462 (1993).

61. M. Giesen-Seibert and H. Ibach, Surf. Sci. 316, 205 (1994); M. Giesen-Seibert, F. Schmitz, R. Jentjens, and H. Ibach, Surf. Sci. 329, 47 (1995).

62. T. J. Stasevich, T.L. Einstein, R. K. P. Zia, M. Giesen, H. Ibach, and F. Szalma, Phys. Rev. B 70, 245404 (2004)

63. Universal connector toy, United States Patent 6179681, now marketed by Superstructs [http://www. superstructs.com/]

64. K. Berland, T.L. Einstein, and P. Hyldgaard, arXiv 0907.0907.4291v1, submitted for publication.

65. D. B. Dougherty, P. Maksymovych, J. Lee, and J. T. Yates, Jr., Phys. Rev. Lett. 97, 236806 (2006)

66. G. Pawin, K.L. Wong, K. Y. Kwon, and L. Bartels, Science 313, 961 (2006).

67. Kwangmoo Kim and T.L. Einstein, unpublished.

68. L. Bartels, T.L. Einstein, et al., unpublished.

69. B.N.J. Persson, Surface Sci. 258, 451 (1991). 